\title{
COMPUTATIONAL TOOLS FOR DESIGNING NI-BASE SUPERALLOYS
}

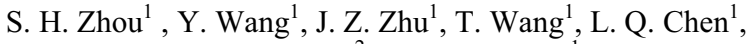 \\ R. A. MacKay ${ }^{2}$, and Zi-Kui Liu ${ }^{1}$ \\ ${ }^{1}$ Department of Materials Science and Engineering, The Pennsylvania State University, University Park, PA 16802. \\ ${ }^{2}$ Materials Division, NASA Glenn Research Center, 21000 Brookpark Road, Cleveland, OH 44135.
}

Keywords: Ni-base Superalloys, Phase-field, First-principles calculations, CALPHAD, Thermodynamic database, Kinetic database, Lattice parameter database.

\begin{abstract}
The main objective of this work is to develop a set of integrated computational tools that can be employed to design Ni-base superalloys. In particular, we developed reliable thermodynamic, kinetic and lattice parameter databases for the Ni-Al-Mo superalloys by combining first-principles calculations and CALPHAD (CALculation of PHAse Diagrams) technique. Microstructural evolution of $\gamma$ precipitate particles was predicted using a quantitative phase-field model that is linked to the thermodynamic, kinetic and lattice parameter databases. Computational results of $\gamma^{\prime}$ coarsening were compared with literature data on particle sizes measured during aging experiments in binary NiAl alloys. The integrated multiscale computational tools have the potential to considerably reduce the time and cost for new superalloy development.
\end{abstract}

\section{Introduction}

The success for designing highly efficient aircraft engines largely depends on our ability to process materials that can withstand the demands of the high temperatures operating environment. A tremendous amount of research has been devoted to finding new Ni-based superalloys, which are thermodynamically and structurally stable at higher temperatures and for longer periods of time. Ni-based superalloys consist of ordered intermetallic $\gamma^{\prime}-\mathrm{L}_{2}$ precipitates embedded in a disordered face-centered cubic $\gamma$-fcc matrix. The control of the $\gamma+\gamma^{\prime}$ two-phase microstructure and its high-temperature stability is the key to the successful development of superalloys with desired high temperature properties since the $\gamma^{\prime}$ precipitate volume fraction, morphology and size distribution in these alloys strongly affect their mechanical properties (e.g. strength, fatigue and creep). The $\gamma$ ' precipitate morphology and spatial distribution are known to depend on a number of factors including the $\gamma$ ' precipitate volume fraction, alloy compositions, temperature, lattice mismatch between the precipitates and matrix, presence of dislocations and applied stress direction. In current laboratory and industrial practice, the traditional trial-and-error method is still the major approach to optimizing the alloy chemistry and processing conditions for achieving desirable microstructures and mechanical properties of Ni-based superalloys. This usually requires many long and expensive experimentations. However, with the rapid development in computer hardware and recent advances in computational modeling, it is now possible to develop a set of computational tools for both fundamental materials research and design of new advanced materials.
The main objective of this work is to combine various computational approaches to determine the relationships between chemistry and microstructure of single-crystal Ni-base superalloys at high temperatures. In section 2 of this paper, the firstprinciples approach is described for calculating the enthalpy of formation of compounds in the ternary Ni-Al-Mo system. This ternary was specifically chosen because of the large amount of experimental data available in the literature on that system. The thermodynamic, kinetic and lattice parameter database developments are presented in sections 3, 4 and 5, respectively, followed by phase-field modeling in section 6 and summary.

\section{First-principles calculations}

First-principles approach has become a powerful tool for calculating the energies of formation at $0 \mathrm{~K}$ for stable and metastable phases. In the present work, the Vienna $a b$ initio simulation package (VASP) ${ }^{1}$ with the ultrasoft pseudopotentials and within the generalized gradient approximation $(\mathrm{GGA})^{2}$ was employed to calculate the energies of formation for a number of selected compounds in the ternary Ni-Al-Mo system. The energy cutoff is determined by the choice of "high accuracy" defined in the VASP software. Monkhost $k$ points were used for the pure elements and the cubic phases and Gamma centered $k$ points were used for the hexagonal phases.

The calculated enthalpies of formation for the binary systems are listed in Table 1 together with the results calculated using the CALPHAD approach. For the Al-Ni and Al-Mo systems, the calculated enthalpies of formation are in reasonable agreement with the CALPHAD results. For the Ni-Mo system, however, the first-principles results indicate that the compounds $\mathrm{Ni}_{2} \mathrm{Mo}$, $\mathrm{Ni}_{3} \mathrm{Mo}, \mathrm{Ni}_{4} \mathrm{Mo}$ and $\mathrm{Ni}_{8} \mathrm{Mo}$ are stable at $0 \mathrm{~K}$ as shown in Figure 1 while the compound $\delta$-NiMo is not stable at $0 \mathrm{~K}$. The dotted-line in Figure 1 is the enthalpy of formation calculated using the model parameters by Cui et al. ${ }^{3}$, which is significantly different from the first-principles results. The improvement of the thermodynamic modeling for the Ni-Mo binary system is thus required, which will be shown in section 3 . 


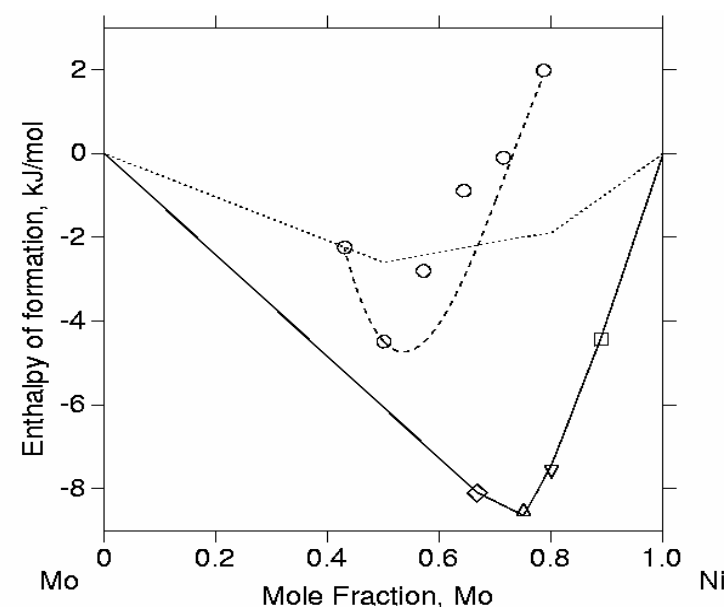

Figure 1. The calculated enthalpy of formation $(-)$ of the binary $\mathrm{Ni}$-Mo system and (---) of the compound $\delta$-NiMo as a function of the composition at $0 \mathrm{~K}$ with the first-principles calculation results $\bigcirc \delta$-NiMo, $\diamond \mathrm{Ni}_{2} \mathrm{Mo}, \triangle \mathrm{Ni}_{3} \mathrm{Mo}, \nabla \mathrm{Ni}_{4} \mathrm{Mo}$, and $\square \mathrm{Ni}_{8} \mathrm{Mo}$ phases and the dotted-line (...) calculated using the parameters by Cui et al. ${ }^{3}$.

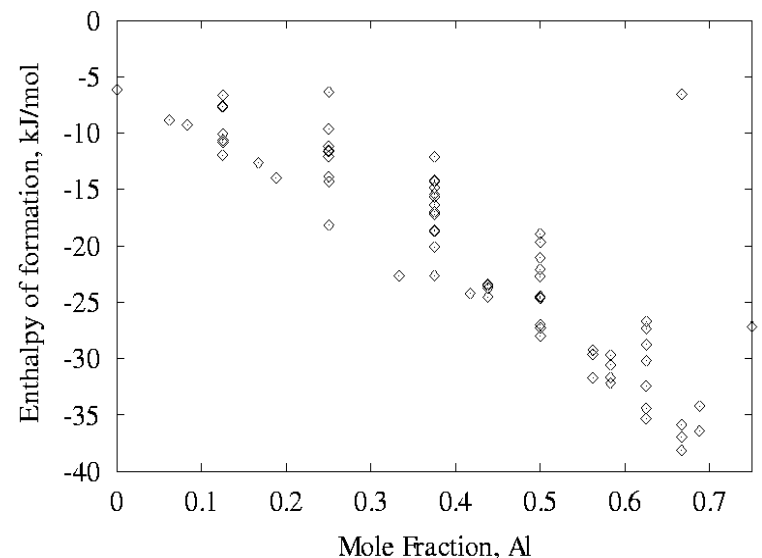

Figure 2. The calculated enthalpy of formation of the compound $\mathrm{N}$ using ATAT and VASP calculation with fixed content $\left(\mathrm{x}_{\mathrm{Mo}}=0.25\right)$ and Mo atomic positions.

Two stable ternary compounds in the ternary Ni-Al-Mo system were reported in the literature ${ }^{4-6}$, i.e. N-phase and X-phase. For the compound $\mathrm{N}$, we limited the starting geometry to the $\mathrm{D}_{22}$ structure with a fixed composition $\left(\mathrm{x}_{\mathrm{Mo}}=0.25\right)$ and fixed positions of Mo atoms. To search for the configurations with the lowest energies, we employed the alloy theoretic automated toolkit (ATAT) developed by van De Walle ${ }^{7}$ combined with VASP. Gamma centered $k$ points was used and more than 65 configurations were studied. Due to the lack of structural information, the first-principles calculation was not performed for the compound $\mathrm{X}$.

The calculated enthalpies of formation for some of the structures found by ATAT are plotted in Figure 2. It is noted that the lowest point along the convex hull of energy is $-38.17 \mathrm{~kJ} / \mathrm{mol}$ located at 66.7 at. $\% \mathrm{Al}$ which coincides with the $\mathrm{Al}_{8} \mathrm{NiMo}_{3}$ stoichiometry. Since the formation energy for $\mathrm{Al}_{8} \mathrm{NiMo}_{3}$ is lower than that of $\mathrm{Al}_{5} \mathrm{NiMo}_{2} \quad(-35.32 \mathrm{~kJ} / \mathrm{mol})$, the proposed stoichiometry of compound $\mathrm{N}$ by Schubert et al. ${ }^{6}$, the stable compound $\mathrm{N}$ is probably $\mathrm{Al}_{8} \mathrm{NiMo}_{3}$ rather than $\mathrm{Al}_{5} \mathrm{NiMo}_{2}{ }^{6}$ and has a body centered orthorhombic unit cell with $b / a=2.322$ and $\mathrm{c} / \mathrm{a}=3.009$ (unit cell with 12 atoms), slightly distorted from those suggested by Schubert et al. ${ }^{6}$ for $\mathrm{Al}_{5} \mathrm{NiMo}_{2}$ with $b / a=2.26$ and $\mathrm{c} / \mathrm{a}=2(\mathrm{c} / \mathrm{a}$ $=3$ if using unit cell of 12 atoms).

\begin{tabular}{|c|c|c|c|c|c|}
\hline \multirow{2}{*}{ Phase } & \multirow{2}{*}{ Structure } & \multirow{2}{*}{$\begin{array}{c}\begin{array}{c}\text { Total } \\
\text { nergy }\end{array} \\
\text { eV/atom }\end{array}$} & \multicolumn{3}{|c|}{ Enthalpy of formation, $\mathrm{kJ} / \mathrm{mol}$} \\
\hline & & & $\begin{array}{c}\text { First- } \\
\text { principles }\end{array}$ & CALP & $\mathrm{AD}$ \\
\hline $\mathrm{Ni}$ & fcc & -5.4827 & 0 & 0 & \multirow{3}{*}{ Ref. $^{8}$} \\
\hline $\mathrm{Al}$ & fcc & -3.6844 & 0 & 0 & \\
\hline Mo & $\mathrm{bcc}$ & -10.827 & 0 & 0 & \\
\hline $\mathrm{Al}_{3} \mathrm{Ni}$ & $\mathrm{D} 0_{11}$ & -4.5597 & -41.07 & -48.48 & \multirow{5}{*}{ Ref. $^{9}$} \\
\hline $\mathrm{Al}_{3} \mathrm{Ni}_{2}$ & $\mathrm{D} 5_{19}$ & -5.0353 & -60.93 & -61.23 & \\
\hline $\mathrm{AlNi}$ & B2 & -5.2459 & -63.90 & -60.83 & \\
\hline $\mathrm{Al}_{3} \mathrm{Ni}_{5}$ & oC16 & -5.3795 & -55.10 & -54.36 & \\
\hline $\mathrm{Ni}_{3} \mathrm{Al}$ & $\mathrm{L1}_{2}$ & -5.4678 & -41.93 & -41.07 & \\
\hline $\mathrm{AlMo}_{3}$ & $\mathrm{Cr}_{3} \mathrm{Si}$ & -9.3130 & -26.20 & -21.44 & \multirow{7}{*}{ Ref. $^{10}$} \\
\hline AlMo & B2 & -7.1948 & 5.88 & -4.19 & \\
\hline $\mathrm{Al}_{8} \mathrm{Mo}_{3}$ & $\mathrm{mC} 22$ & -5.9881 & -34.31 & -37.50 & \\
\hline $\mathrm{Al}_{3} \mathrm{Mo}$ & $\mathrm{mC} 32$ & -5.8119 & -32.98 & -32.00 & \\
\hline $\mathrm{Al}_{4} \mathrm{Mo}$ & $\mathrm{Al}_{4} \mathrm{~W}$ & -5.3931 & -27.03 & -27.51 & \\
\hline $\mathrm{Al}_{5} \mathrm{Mo}$ & $\mathrm{Al}_{5} \mathrm{~W}$ & -5.1330 & -24.90 & -23.18 & \\
\hline $\mathrm{Al}_{12} \mathrm{Mo}$ & $\mathrm{Al}_{12} \mathrm{~W}$ & -4.3725 & -13.37 & -10.7 & \\
\hline NiMo & $\mathrm{P} 2_{1} 2_{1} 2_{1}$ & -8.2014 & -4.49 & -4.49 & \multirow{5}{*}{ Ref. $^{11}$} \\
\hline $\mathrm{Ni}_{2} \mathrm{Mo}$ & $\mathrm{Pt}_{2} \mathrm{Mo}$ & -7.3507 & -8.35 & -8.09 & \\
\hline $\mathrm{Ni}_{3} \mathrm{Mo}$ & $\mathrm{D} 0_{\mathrm{a}}$ & -6.9073 & -8.54 & -8.63 & \\
\hline $\mathrm{Ni}_{4} \mathrm{Mo}$ & $\mathrm{D} 1_{\mathrm{a}}$ & -6.6298 & -7.55 & -7.43 & \\
\hline $\mathrm{Ni}_{8} \mathrm{Mo}$ & $\mathrm{Ni}_{8} \mathrm{Nb}$ & -6.1225 & -4.44 & -4.38 & \\
\hline
\end{tabular}

\section{Thermodynamic database development}

The ternary Ni-Al-Mo system consists of three pure elements and three binary systems. The Gibbs energy functions of the pure elements were taken from database by Dinsdale ${ }^{8}$. The thermodynamic database of the binary Ni-Al ${ }^{9}$ and Al-Mo ${ }^{10}$ systems in the literature were used in the evaluation of the ternary $\mathrm{Ni}-\mathrm{Al}-\mathrm{Mo}$ system. For the binary Ni-Mo and ternary Ni-Al-Mo systems, the Gibbs energy functions of individual phases were evaluated with the first-principles calculation data and the experimental data in the literature using the Parrot module ${ }^{12}$ in Thermo-Calc ${ }^{13}$. The calculated phase diagram of the binary NiMo system is shown in Figure 3. The calculated enthalpies of formation are plotted in Figure 1 and also listed in Table 1, which show good agreement with the results from first-principles calculations. The evaluation of the binary Ni-Mo system was discussed in detail in reference ${ }^{11}$.

The first-principles calculated enthalpy of formation for $\mathrm{Al}_{8} \mathrm{NiMo}_{3}$ with the slightly distorted $\mathrm{DO}_{22}$ structure is $38.17 \mathrm{~kJ} / \mathrm{mol}$ at $0 \mathrm{~K}$, while the enthalpy of mixing of the $\mathrm{Al}_{8} \mathrm{Mo}$, $\mathrm{AlMo}_{3}$ and $\mathrm{B}_{2}$ phases is $-41.49 \mathrm{~kJ} / \mathrm{mol}$ and $-40.62 \mathrm{~kJ} / \mathrm{mol}$ calculated by CALPHAD and the first-principles approaches, respectively. This indicates that $\mathrm{Al}_{8} \mathrm{NiMo}_{3}$ is not stable at $0 \mathrm{~K}$. 


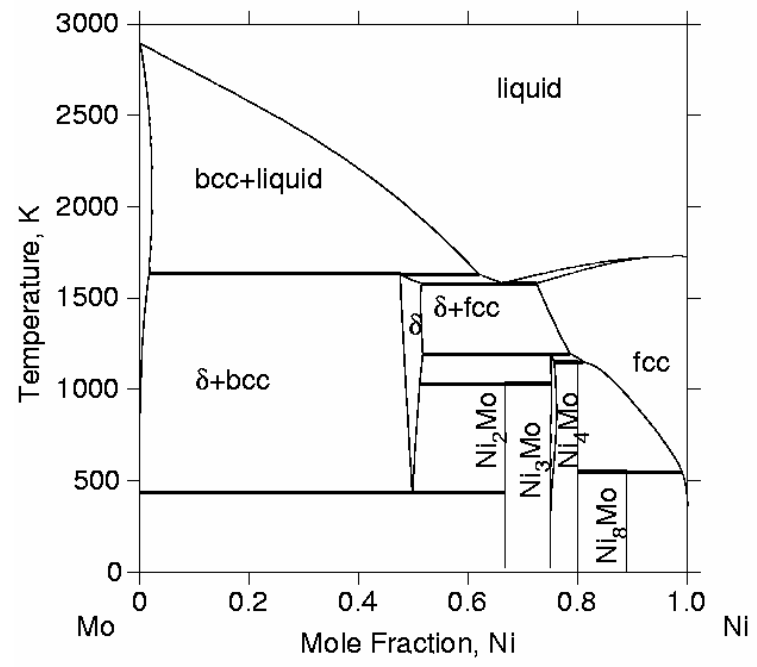

Figure 3. The binary Ni-Mo phase diagram calculated using the parameters by Zhou et al. ${ }^{11}$.

A typical microstructure of the Ni-base alloys consists of the dispersion of ordered intermetallic precipitate $\gamma$, particles embedded in the $\gamma$ matrix. Thermodynamic properties of the $\gamma$ ' and $\gamma$ phases provide the information for the phase equilibria, phase stability, and driving force for internal processes under various conditions, which is critical for the phase-field modeling. Thus, the evaluation of the Gibbs energy functions of $\gamma$ ', $\gamma$ and other phases are required. In this work, the thermodynamics of $\gamma$ and $\gamma$ ' phases were modeled with a single Gibbs energy function taking into account the crystallographic relation between the two phases $^{9,14}$. For the $\gamma$ phase, its Gibbs energy has following formula:

$$
G_{m}^{\gamma}=\sum_{i} x_{i}{ }^{\circ} G_{i}^{\gamma}+R T\left(\sum_{i} x_{i} \ln x_{i}\right)+{ }^{x s} G_{m}^{\gamma} \quad(i=A l, N i, M o)
$$

where $R$ is the gas constant, ${ }^{\circ} G_{i}^{\gamma}$ the molar Gibbs energy of the pure element $i$ by Dinsdale ${ }^{8}$. The second term is the ideal Gibbs energy of mixing. ${ }^{x s} G_{m}^{\gamma}$ is the excess Gibbs energy. For the $\gamma^{\prime}$ phase, its Gibbs energy function is given as:

$$
\begin{aligned}
G_{m}^{\gamma^{\prime}}= & G_{m}^{\gamma}\left(x_{i}\right)+\Delta G_{m}^{o r d}\left(y_{i}^{I}, y_{i}^{I I}\right) \\
& =G_{m}^{\gamma}\left(x_{i}\right)+G_{m}^{o r d}\left(y_{i}^{I}, y_{i}^{I I}\right)-G_{m}^{o r d}\left(x_{i}, x_{i}\right)
\end{aligned}
$$

where $y_{i}^{I}$ and $y_{i}^{I I}$ are the site fractions of the element $i$ in the first and second sublattices. $G_{m}^{\gamma}\left(x_{i}\right)$ corresponds to the Gibbs energy of the $\gamma$-fcc phase in equation $1 . \Delta G_{m}^{\text {ord }}\left(y_{i}^{I}, y_{i}^{I I}\right)$ is the ordering energy, which is the difference between $G_{m}^{\text {ord }}\left(y_{i}^{I}, y_{i}^{I I}\right)$ and $G_{m}^{\text {ord }}\left(x_{i}, x_{i}\right)^{9,}{ }^{18} . G_{m}^{\text {ord }}\left(y_{i}^{I}, y_{i}^{I I}\right)$ and $G_{m}^{\text {ord }}\left(x_{i}, x_{i}\right)$ are calculated using the same function but for the ordered and disordered states, respectively. With the experimental data in the literature supplemented by results from the first-principles calculations, the Gibbs energy functions of individual phases in the ternary Ni-Al-Mo system were evaluated as discussed in details in reference ${ }^{19}$. The calculated isothermal section at $1473 \mathrm{~K}$ is shown in Figure 4, in which the experimental data ${ }^{4,15-17}$ are well reproduced.

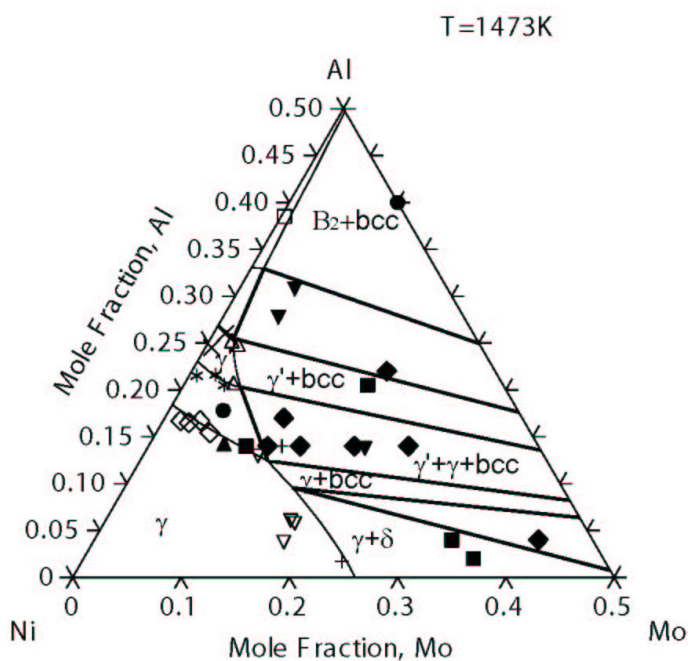

Figure 4. The calculated isothermal sections at $1473 \mathrm{~K}$ in comparison with experimental data $+^{4}, \nabla^{15}, \diamond{ }^{16}$ phase boundary of the $\gamma$ phase; $\times^{4}, \square^{15}, *^{16}$ phase boundary of the $\gamma^{\prime}$ phase; $\boldsymbol{\Delta}^{15}$ single phase field; $\boldsymbol{\square}^{15}, \boldsymbol{}^{17}$ two phase field; $\boldsymbol{}^{15}, \nabla^{17}$ three phase field; $\triangle^{15}$ phase boundary of the $\mathrm{B}_{2}$ phase.

\section{Kinetic database development}

The third step for the computational tools for designing Ni-based superalloys is to develop the kinetic and lattice parameter databases. Kinetic database is based on the mobility descriptions of all components in different phases, and the diffusion mobility $M_{i}$ is modeled by following equations ${ }^{20}$ :

$$
\begin{aligned}
M_{i}= & \frac{1}{R T} \exp \left(\frac{-\Delta G_{i}}{R T}\right) \\
\Delta G_{i}= & \sum_{j} \sum_{m} y_{j}^{I} y_{m}^{I I} \Delta G_{i}^{j: m}+\sum_{j} \sum_{k>j} \sum_{m} y_{j}^{I} y_{k}^{I} y_{m}^{I I} \Delta G_{i}^{j, k: m}+ \\
& \sum_{j} \sum_{n} \sum_{m>n} y_{j}^{I} y_{n}^{I I} y_{m}^{I I} \Delta G_{i}^{j: n, m}
\end{aligned}
$$

where $\Delta G_{i}$ is the activation Gibbs energy of component $i . \Delta G_{i}^{j: m}$ is the activation Gibbs energy of component $i$ with components $\mathrm{j}$ and $m$ on the first and second sublattices. $\Delta G_{i}^{j, k: m}$ and $\Delta G_{i}^{j: n, m}$ are the interaction terms.

Based on the generalized Onsager flux equation ${ }^{20,21}$, various diffusivities can be calculated by the thermodynamic database and diffusion mobility database. For example, the intrinsic diffusion coefficient, $D_{k j}$ (the diffusivity of component $k$ with respect to the gradient of component $k$ ), can be expressed as:

$$
D_{k j}=\sum_{i \in S}\left(\delta_{i k}-u_{k}\right) u_{i} M_{i} \frac{\partial \mu_{i}}{\partial u_{j}}+\sum_{i \notin S} \delta_{i k} u_{i} y_{V a} M_{i} \frac{\partial \mu_{i}}{\partial u_{j}}
$$

where $u_{k}=x_{k} / \sum_{i \in S} x_{i}, \delta_{i k}$ is the Kronecker-delta function, $i \in S$ denotes that component $i$ is substitutional and $i \notin S$ interstitial. $x_{k}$ is the mole fraction of component $k . \mu_{i}$ is the 
chemical potential of component $i$ calculated from the thermodynamic database as discussed in section 3. $y_{V a}$ represents the site fraction of vacancy in the interstitial sublattice.

Similar to the thermodynamic database development, the parameters describing the diffusion mobilities are extracted from various experimental data, especially from the chemical and the tracer diffusion coefficients. A detailed description of the optimization procedure is referred to in reference ${ }^{20}$.

The previously assessed diffusion mobilities in Ni-Al system by Engstrom and Agren ${ }^{22}$ are employed in the present work. Based on the work by Campbell et al. ${ }^{23}$ and additional experimental data, the diffusion mobilities in the Ni-Mo system were re-evaluated in the present work. For Al-Mo system, because of very limited solubility range and experimental data, no interaction parameters were adopted.

By combining the above mobility descriptions of binary systems, kinetic modeling of the Ni-Al-Mo ternary can be carried out in a similar procedure. Since no experimental data was available on the ternary alloy, no high-order interaction parameters are used in present work.

\section{Lattice parameter database development}

A phenomenological modeling of the composition and temperature dependent lattice parameters in solid states has been recently developed by present authors ${ }^{24}$, and used to construct the lattice parameter database for the fcc phase in Ni-base superalloys.

Lattice parameter modeling begins with the evaluation of the thermal expansion coefficients of pure elements, which are then used in calculation of their temperature-dependent lattice parameters. Similar to the Gibbs energy modeling in CALPHAD, the lattice parameter is related to composition with the following equations:

$$
\begin{aligned}
& a=\sum_{i} x_{i}{ }^{0} a_{i}+{ }^{e x} a \\
& { }^{e x} a=\sum_{i} \sum_{j>i} x_{i} x_{j} \sum_{k=0}^{n}{ }^{k} I_{i, j}\left(x_{i}-x_{j}\right)^{k}
\end{aligned}
$$

where ${ }^{0} a_{i}$ denotes the lattice parameter of component $i,{ }^{e x} a$ is the excess contribution, and ${ }^{k} I_{i, j}$ describe the interaction between components $i$ and $j$.

The model parameters can be evaluated from the available experimental data. Figure 5 shows the lattice parameter of the fcc phase in the Ni-Al system calculated as a function of composition at $298 \mathrm{~K}$. Since there are no reliable experimental data available in the Al-Mo system, the VASP calculations based on the GGA potentials have been used to determine the interaction parameters. In addition, results from first-principle calculations together with experimental data were also used for evaluating the lattice parameters of the Ni-Mo system.

By combining the evaluated constitutive binary systems and ternary experimental data, the lattice parameters of fcc phase in Ni-Al-Mo ternary system are obtained by a similar approach. The lattice parameter database of Ni-Al-Mo alloys developed covers the whole composition and temperature range, including experimentally uninvestigated regions.

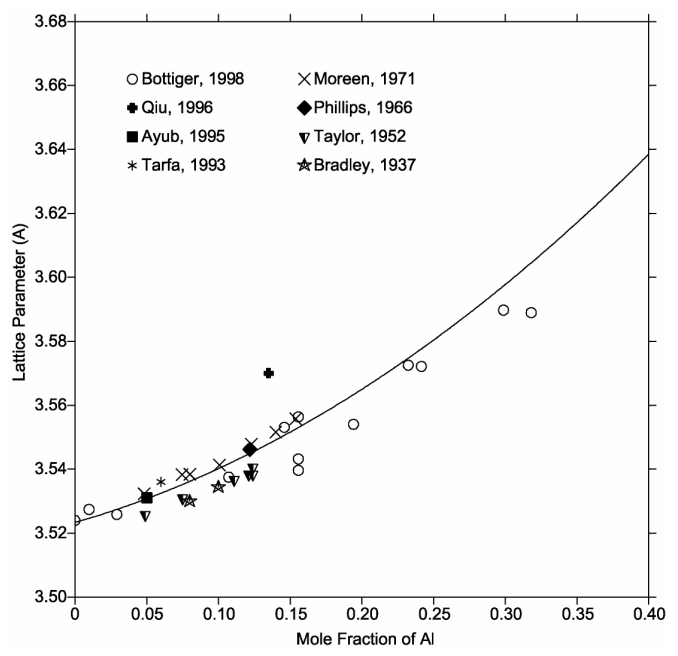

Figure 5. The calculated lattice parameter of the fcc phase in the $\mathrm{Ni}-\mathrm{Al}$ system at $298 \mathrm{~K}$ with experimental data ${ }^{25-32}$.

\section{Phase-field simulations}

The final step is phase-field simulation. A recent important development in computational materials science is the emergence of the powerful phase-field approach ${ }^{33}$ for modeling phase transformations and microstructure evolution based on the thermodynamic, kinetic and lattice parameter databases. One of the main advantages of phase-field approach is that the temporal evolution of any arbitrary microstructures can be predicted without any a priori assumptions about their evolution path. For simplicity, we consider a binary $\mathrm{Ni}-\mathrm{Al}$ alloy as an example in following discussions. The model described below for binary Ni$\mathrm{Al}$ alloys is being expanded to the ternary and multi-component systems.

The phase-field approach describes a microstructure by using a set of field variables. To distinguish the four types of ordered domains of the $\gamma^{\prime}$ phase and the disordered $\gamma$ phase in a binary NiAl alloy, we employed one composition field $\mathrm{c}(\mathrm{r}, \mathrm{t})$ and four artificial order parameter fields $\phi_{\mathrm{i}}(\mathrm{r}, \mathrm{t})(\mathrm{i}=1,2,3,4)$ to describe the $\gamma+\gamma^{\prime}$ two-phase microstructure. The $\gamma / \gamma^{\prime}$ interfaces are represented by diffuse regions with a certain thickness where the phase-field variables change continuously. The total free energy of an inhomogeneous microstructure described by the field variables is then given ${ }^{34}$

$$
F=\int_{r}\left[f\left(c, \phi_{i}\right)+\frac{\varepsilon^{2}}{2} \sum_{i}\left(\nabla \phi_{i}\right)^{2}\right] d r
$$

where $\varepsilon^{2}$ is the gradient energy coefficient of the order parameters. The diffuse-interface region is regarded as a mixture of the precipitate phase and matrix phases with different compositions but with equal chemical potentials ${ }^{35}$ 


$$
c=h\left(\phi_{i}\right) c^{p}+\left(1-h\left(\phi_{i}\right)\right) c^{m}, \quad \frac{\partial f^{p}}{\partial c^{p}}=\frac{\partial f^{m}}{\partial c^{m}}
$$

where $\mathrm{h}\left(\phi_{\mathrm{i}}\right)$ is a continuous function with values between 0 and 1 . The chemical free energies of the precipitate and matrix phases, $\mathrm{f}^{\mathrm{p}}$ and $\mathrm{f}^{\mathrm{m}}$, were taken directly from the thermodynamic database described in section 3. For given composition $\mathrm{c}$ and order parameters $\phi_{i}$, there exists a single solution pair of $c^{p}$ and $c^{m}$ that satisfies Eq. (9). The local free energy density of the system $\mathrm{f}(\mathrm{c}$, $\left.\phi_{\mathrm{i}}\right)$ is written as

$$
\begin{aligned}
f\left(c, \phi_{i}\right)= & h\left(\phi_{i}\right) f^{p}\left(c^{p}\right)+\left(1-h\left(\phi_{i}\right)\right) f^{m}\left(c^{m}\right) \\
& +w g\left(\phi_{i}\right)+f^{e l}\left(\phi_{i}\right)
\end{aligned}
$$

where $g\left(\phi_{i}\right)$ is the double-well potential, $w$ the double-well potential height, and $f^{e l}$ the elastic energy density. The elastic energy contribution to the morphological evolution and coarsening kinetics of $\gamma^{\prime}$ particles, $f^{l}$, can be obtained using the microelasticity theory ${ }^{36}$.

The morphological evolution of $\gamma^{\prime}$ precipitates is obtained by solving the two sets of kinetic equations ${ }^{34,35}$ :

$$
\begin{aligned}
& \frac{\partial \phi_{i}}{\partial t}=-L \frac{\delta F}{\delta \phi_{i}} \\
& \frac{\partial c}{\partial t}=\nabla \cdot\left[M \nabla \frac{\delta F}{\delta c}\right]
\end{aligned}
$$

where $t$ is the time, $M$ the diffusion mobility, and $L$ the kinetic coefficient for order parameter relaxation. The diffusion mobility term $M$ is related to the atomic mobilities of $\mathrm{Ni}$ and $\mathrm{Al}$, which are obtained from the kinetic database as discussed in section 4 . The value of $L$ is selected to ensure that the $\gamma^{\prime}$ precipitate evolution process is diffusion-controlled. Nucleation of $\gamma^{\prime}$ from the supersaturated $\gamma$ phase was explicitly introduced into the system at the beginning of a phase-field simulation. Periodic boundary conditions were assumed in the computational domain. A semiimplicit Fourier spectral method was used for numerically solving these two sets of equations ${ }^{37,38}$.

In our phase-field simulations, almost all the required modeling parameters are either taken from databases or obtained from experimental measurements. The Gibbs free energy and diffusion mobility were imported from our thermodynamic and kinetic databases. Lattice mismatch was obtained from the lattice parameter database. Experimentally measured values used in the simulation also include the interfacial energy and elastic constants. The only disposable quantitative parameter is the diffusive thickness of $\gamma / \gamma^{\prime}$, which determines the unit grid length scale and correspondingly the maximum system size that can be handled. The unit time step is chosen to maintain numerical accuracy and stability. For a given temperature and alloy composition, the morphological evolution pattern can be simulated with real length and time scales. Quantitative information, such as the coarsening rate constant and particle size distribution, can also be analyzed from the output of the field variable distributions.
Figure 6 shows the 3D morphological evolution of $\gamma$ ' precipitates in a Ni-13.8 at.\%Al alloy at a temperature of $1023 \mathrm{~K}$. The initial state was a homogeneous solution with small composition fluctuations around the average composition. The volume fraction of the $\gamma^{\prime}$ phase at the four different aging times in Figure 6 is approximately $9-12 \%$, which is very close to the equilibrium volume fraction of $10 \%$. Precipitate coarsening is clearly seen in Figure 6 where the number of particles in the computational domain decreases from about 2500 at $\mathrm{t}=15 \mathrm{~min}$ to 110 at $\mathrm{t}=8 \mathrm{hr}$. Shrinking and growth of precipitates, as well as the coalescence of two neighboring domains, are observed. Driven by the reduction of the interfacial energy and elastic energy, the average precipitate size grows, accompanied by a change of particle shape from spherical to cuboidal, rod-like, or plate-like at later stages. The shape change from spheres to cubes occurs when the average particle size is around $20-25 \mathrm{~nm}$ at an aging time about $2-4 \mathrm{hr}$, which is in good agreement with experimental observations ${ }^{39}$.

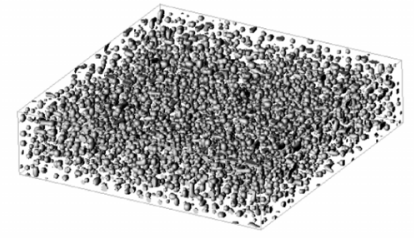

(a)

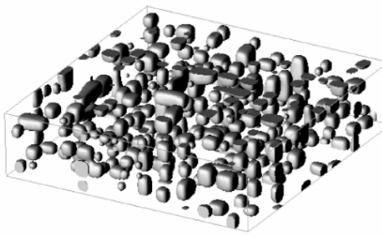

(c)

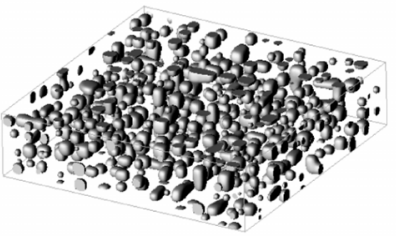

(b)

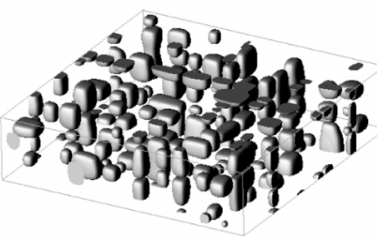

(d)
Figure 6. Morphological evolution of $\gamma^{\prime}$ precipitates from 3D simulations in a Ni-13.8at\%Al alloy aged at $1023 \mathrm{~K}$ (a) $\mathrm{t}=15$ min; (b) $\mathrm{t}=2 \mathrm{hr}$; (c) $\mathrm{t}=4 \mathrm{hr}$; (d) $\mathrm{t}=8 \mathrm{hr}$. Computational.

A comparison of the average $\gamma^{\prime}$ particle size evolution obtained from phase-field simulations and experiments ${ }^{39}$ is shown in Fig. 7 by plotting $\bar{a}^{3}-\bar{a}_{0}^{3}$ versus $\mathrm{t}$ in a logarithmic scale, where $\bar{a}$ is the average cube edge length, $\mathrm{t}$ is the aging time, and $\bar{a}_{0}$ is a constant related to the average particle size at the beginning of coarsening. In this figure, the rate constant, or coarsening rate, can be evaluated from the intercept with any line parallel to the $\bar{a}^{3}-\bar{a}_{0}^{3}$ axis. While the cubic growth law is observed in both simulations and experiments, the coarsening rate determined from simulation results was approximately $20 \%$ higher than the experimentally determined value. With all possible errors considered including the inaccuracy of simulation parameters such as the interfacial energy, diffusion mobility and lattice mismatch, and systematic errors in the experimental measurements, the agreement of average particle size evolution obtained from simulations and experiments is excellent. 


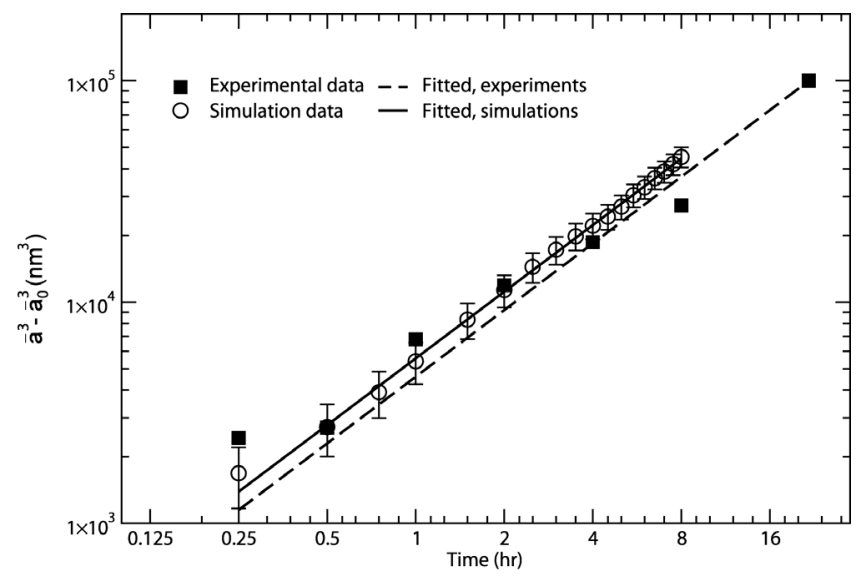

Figure 7. Average particle size as a function of time plotted as $\bar{a}^{3}-\bar{a}_{0}^{3}$ versus $\mathrm{t}$ in a $\mathrm{Ni}-13.8 \mathrm{at} \% \mathrm{Al}$ alloy aged at $1023 \mathrm{~K}$, experimental data and the fit of experimental data from reference ${ }^{39}$.

\section{Summary}

A set of integrated computational tools was developed successfully to predict the relationships between the chemistry and microstructure for Ni-Al binary and Ni-Al-Mo ternary alloys. The thermodynamic, kinetic and lattice parameter databases were developed with the first-principles calculation results and the experimental data in the literature. The microstructures of $\gamma$ and $\gamma$ ' phases were modeled using phase-field technique with an interface to the thermodynamic and kinetic databases. The calculated results on $\gamma^{\prime}$ coarsening are in good agreement with existing experimental data on binary alloys. The computational tools can be extended to multicomponent Ni-base superalloys.

\section{Acknowledgement}

This work is supported by the Ultra Efficient Engine Technology Program at NASA Glenn Research Center under grant NCC3-920. We are also grateful for the computing resource award from NCSA under grant no. DMR030004N and DMR020002P. Simulations were performed in Pittsburgh Supercomputing Center and the LIONXL linux cluster supported partly by NSF grants under no. DMR-0205232, DMR-0122638, DMR-9983532 and DMR-9633719, as well as the Materials Simulation Center, a Penn-State MRSEC and MRI facility.

\section{G. Kresse, T. Demuth and F. Mittendorfer,} VAMP/VASP, http://cms.mpi.univie.ac.at/vasp/, 2003.

2. J. P. Perdew and Y. Wang, "Accurate and Simple Analytic Representation of the Electron-Gas Correlation-Energy," Phys. Rev. B, 45 (1992) 13244-13249.

3. Y. Cui, Z. Jin and X. Lu, "Experimental study and thermodynamic assessment of the Ni-Mo-Ta ternary system*," Met. Mater. Trans. A, 30A (1999) 2735-2744.

4. V. Y. Markiv, V. V. Burnashova, L. I. Pryakhina and K. P. Myasnikova, "Phase Equilibria in the Mo-Ni-Al System," Izv. Akad Nauk SSSR Metall., 5 (1969) 180-185.
5. B. Grushko, S. Mi and J. G. Highfield, "A study of the Al-rich region of the Al-Ni-Mo alloy system," J. Alloy. Compd., 334 (2002) 187-191.

6. K. Schubert, A. Raman and W. Rossteutscher, "Einige Strukturdaten Metallischer phasen (X)," Naturwissenschaften, 51 (1964) 506-507.

7. A. van de Walle, M. Asta and G. Ceder, "The Alloy Theoretic Automated Toolkit: A user guide," CALPHAD, 26 (2002) 539-553.

8. $\quad$ A. T. Dinsdale, "SGTE Data for Pure Elements," CALPHAD, 15 (1991) 317-425.

9. N. Dupin, I. Ansara and B. Sundman, "Thermodynamic re-assessment of the ternary system Al-Cr-Ni," CALPHAD, 25 (2001) 279-298.

$10 . \quad$ N. Saunders, "The Al-Mo system (aluminummolybdenum)," J. Phase Equilib., 18 (1997) 370-378.

11. S. H. Zhou, Y. Wang, C. Jiang, J. Z. Zhu, R. A.

MacKay, L. Q. Chen and Z. K. Liu, "Evaluation of the Thermodynamic Properties of the Ni-Mo System with FirstPrinciples Calculation Data," To be published, (2004)

12. B. Jansson, Evaluation of Paramters in Thermochemical Models Using Different Types of Experimental Data Simultaneously,(Stockholm, Sweden, Royal Institute of Technology, 1984).

13. J. O. Andersson, T. Helander, L. H. Hoglund, P. F. Shi and B. Sundman, "THERMO-CALC \& DICTRA, computational tools for materials science," CALPHAD, 26 (2002) 273-312.

14. N. Dupin and B. Sundman, "A thermodynamic database for Ni-base superalloys," Scand. J. Metall., 30 (2001) 184-192.

15. S. B. Maslenkov, N. N. Burova and V. A. Rodimkina, "Ni--NiAl--Mo Phase Diagram in the 1200-700 deg C Temperature Range," Russ. Metall., (1988) 179-185.

16. C. C. Jia, K. Ishida and T. Nishizawa, "Partition of Alloying Elements between Gamma-(A1), Gamma(')-(L12), and Beta-(B2) Phases in Ni-Al Base Systems," Metall. Mater. Trans. $A, 25$ (1994) 473-485.

17. P. Nash, S. Fielding and D. R. F. West, "Phase Equilibria in Nickel Rich Ni--Al--Mo and Ni--Al--W Alloys," Met. Sci., 17 (1983) 192-194.

18. B. Sundman, I. Ansara, M. Hillert, G. Inden, H. L. Lukas and K. C. H. Kumar, "Contributions to the thermodynamic modelling of solutions," Z. Metallk., 92 (2001) 526-532.

19 S. H. Zhou, Y. Wang, C. Jiang, J. Z. Zhu, R. A.

MacKay, L. Q. Chen and Z. K. Liu, "Evaluation of the Thermodynamic Properties of the Ni-Al-Mo System with FirstPrinciples Calculation Data," To be published, (2004)

$20 . \quad$ B. Jonsson, "Assessment of the Mobilities of $\mathrm{Cr}, \mathrm{Fe}$, and $\mathrm{Ni}$ in Fcc Cr-Fe-Ni Alloys," Z. Metallkd., 86 (1995) 686-692.

21. J.-O. Andersson, L. Hoglund, B. Jonsson and J. Agren, Computer Simulation of Multicomponent Diffusional Transformations in Steel,(Pergmon Press, 1990).

22. A. Engstrom and J. Agren, "Assessment of diffusional mobilities in face-centered cubic Ni Cr Al alloys," Z. Metallkd., 87 (1996) 92-97.

23. C. E. Campbell, W. J. Boettinger and U. R. Kattner, "Development of a Diffusion Mobility Database for Ni-base Superalloys," Acta Mater., 50 (2002) 775-792.

24. T. Wang, J. Z. Zhu, R. A. MacKay, L. Q. Chen and Z. K. Liu, "Modeling of Lattice Parameter in Ni-Al System," in submission, (2004)

25. G. Ayub, F. A. Khwaja, A. U. Haq and Z. Ahmad, "Ordering Behavior of Ni-5 at-Percent Al-Alloy," Acta Metall. Mater., 43 (1995) 1457-1465. 
26. J. Bottiger, N. Karpe, J. P. Krog and A. V. Ruban,

"Measured and calculated thermoelastic properties of

supersaturated fcc $\mathrm{Ni}(\mathrm{Al})$ and $\mathrm{Ni}(\mathrm{Zr})$ solid solutions," J. Mater.

Res., 13 (1998) 1717-1723.

27. A. J. Bradley and A. Taylor, "X-ray Analysis of the

Nickel-Aluminum System," Proc. Roy. Soc., A159 (1937)

28. H. A. Moreen, R. Taggart and D. H. Polonis, "Model for the Prediction of Lattice Parameters of Solid Solutions," Metall.

Trans., 2 (1971) 265-268.

29. V. A. Phillips, "A Metallographic Study of Precipitation in a Ni-12.7 At. Per Cent Al Alloy," Acta Mater., 14 (1966) 15331547.

30. Y. Y. Qiu, "Coarsening kinetics of gamma' precipitates in Ni-Al and Ni-Al- Mo alloys," J. Mater. Sci., 31 (1996) 4311-

4319.

31. T. Tarfa, B. Sitaud and O. Dimitrov, "The Effects of Ti or Ta on the Kinetics of Short-Range Ordering and Self-Diffusion in Ni(Al)-Based Solid-Solutions," Acta Metall. Mater., 41 (1993) 3191-3202.

32. A. Taylor and R. W. Floyd, "The Constitution of Nickel-rich Alloys of the Nickel-Titanium-Aluminium System," J. Inst. Metal., 81 (1952-1953) 25-32.

33. L. Q. Chen, Annu. Rev. Mater. Res., 32 (2002) 113.

34. J. W. Cahn and J. E. Hilliard, J. Chem. Phys., 28 (1958)

258.

35. S. G. Kim, W. T. Kim and T. Suzuki, "Phase-field model for binary alloys," Phys. Rev. E, 60 (1999) 7186-7197. 36. A. G. Khachaturyan, Theory of Structural

Transformation in Solids,(New York, Wiley, 1983).

37. L. Q. Chen and J. Shen, "Applications of semi-implicit Fourier-spectral method to phase field equations," Comput. Phys. Commun., 108 (1998) 147-158.

38. J. Z. Zhu, L. Q. Chen, J. Shen and V. Tikare, "Coarsening kinetics from a variable-mobility Cahn-Hilliard equation: Application of a semi-implicit Fourier spectral method," Phys. Rev. E, 60 (1999) 3564-3572.

39. A. J. Ardell and R. B. Nicholson, J. Phys. Chem. Solids, 27 (1966) 1793. 
